\title{
Inherent bias of SARS-CoV-2 RNA quantification for wastewater surveillance due to variable RT-qPCR assay parameters
}

Aaron Bivins $^{1}$, Devrim Kaya ${ }^{2}$, Kyle Bibby ${ }^{1}$, Stuart L. Simpson ${ }^{3}$, Stephen A. Bustin ${ }^{4}$, Orin C.

Shanks ${ }^{5}$, Warish Ahmed ${ }^{6 *}$

${ }^{1}$ Department of Civil \& Environmental Engineering \& Earth Sciences, University of Notre

Dame, Notre Dame, IN 46556.

${ }^{2}$ School of Chemical, Biological, \& Environmental Engineering, Oregon State University,

Corvallis, OR 97331.

${ }^{3}$ CSIRO Land and Water, Lucas Heights, NSW 2234, Australia.

${ }^{4}$ Medical Technology Research Center, Faculty of Health, Education and Social Care, Anglia

Ruskin University, Chelmsford, Essex, CM1 1SQ, UK.

${ }^{5}$ United States Environmental Protection Agency, Office of Research and Development, 26W

Martin Luther King Jr. Drive, Cincinnati, OH, 45268, USA.

${ }^{6}$ CSIRO Land and Water, Ecosciences Precinct, 41 Boggo Road, Dutton Park 4102, QLD,

Australia.

*Corresponding author. Warish Ahmed. Mailing address: Ecosciences Precinct, 41 Boggo Road,

Dutton Park 4102, Queensland, Australia Tel.: +617 3833 5582; E-mail address:

Warish.Ahmed@csiro.au 


\begin{abstract}
The coronavirus disease 2019 (COVID-19) pandemic has led to wastewater surveillance becoming an important tool for monitoring the spread of severe acute respiratory syndrome coronavirus 2 (SARS-CoV-2) within communities. As a result, molecular methods, in particular reverse transcription-quantitative PCR (RT-qPCR), have been employed to generate large data sets aimed at the detection and quantification of SARS-CoV-2 in wastewater. Although RTqPCR is rapid and sensitive, there is no standard method that fits all use cases, there are no certified quantification standards and experiments are carried out using numerous different assays, reagents, instruments, and data analysis protocols. These variations can lead to the reporting of erroneous quantitative data resulting in potentially misleading interpretations and conclusions. We have reviewed the SARS-CoV-2 wastewater surveillance literature focusing on variability of RT-qPCR data as revealed by inconsistent standard curves and associated parameters. We find that variation in these parameters and deviations from best practices as described in The Minimum Information for Publication of Quantitative Real-Time PCR Experiments (MIQE) guidelines suggest a lack of reproducibility and reliability in quantitative measurements of SARS-CoV-2 RNA in wastewater.
\end{abstract}

Keywords: RT-qPCR, assay validity, standard curve, quality assurance, quality control, wastewater surveillance. 


\section{Introduction}

The screening of untreated wastewater and primary solids for severe acute respiratory syndrome coronavirus 2 (SARS-CoV-2) RNA has emerged as an effective means of tracking coronavirus disease 2019 (COVID-19) at a population level (Ahmed et al., 2020a; Medema et al., 2020a; Peccia et al., 2020; D’Aoust et al., 2021; Li et al., 2021). SARS-CoV-2 RNA wastewater surveillance is being employed as a tool to monitor COVID-19 alongside traditional clinical monitoring in $>50$ countries across hundreds of organizations and sites (https://arcg.is/1aummW; Bivins et al., 2020; Naughton et al., 2021). However, SARS-CoV-2 RNA surveillance in wastewater is a complex process that involves a sequence of steps including wastewater sampling, transport, virus concentration (if required), RNA extraction, reverse-transcription quantitative PCR assays (RT-qPCR) and data interpretation (Ahmed et al. 2021). Unless validated and optimized, these steps, individually or collectively, can produce erroneous results, which may lead to inaccurate evaluations of COVID-19 status within communities. This is particularly important for reporting quantitative data, rather than qualitative detected/not detected results. While recent reviews have highlighted the limitations and bottlenecks of wastewater surveillance from sampling to SARS-CoV-2 detection and quantification (Medema et al., 2020b; Michael-Kordatou et al., 2020; Ahmed et al. 2021, Zhu et al., 2021), little attention has been paid to the reliability, accuracy and relevance of quantitative data generated by RT-qPCR-based wastewater testing.

Quantification by RT-qPCR is based on the quantification cycle $(\mathrm{Cq})$, which is usually obtained by defining a baseline cycle range below which amplification is not recorded and uses a multiple of the standard deviation of the noise levels in the baseline to establish a threshold line which, upon crossing the amplification curves, generates $\mathrm{Cq}$ values. The $\mathrm{Cq}$ is inversely proportional to the initial template concentration, and samples are quantified by comparing their 
$\mathrm{Cq}$ values relative to assay-specific standard curves. These are constructed using a dilution series of standard control materials, typically four to six 10- fold dilution points, with a defined number of target sequences.

The standard curve is created by plotting the resultant $\mathrm{Cq}$ values against log transformed gene copy (GC) quantities from control materials and fitting a linear trend line to the data $(\mathrm{y}=\mathrm{mx}+$ b). The precision of the quantitative data is strongly influenced by the quality of the standard curve as reflected by its slope (m), PCR efficiency, linearity ( $r^{2}$ value) and y-intercept (b). PCR efficiency is calculated from the slope $(\mathrm{m})$ of the trendline, with an optimal efficiency, resulting in a doubling of the PCR product after each cycle, characterized by a slope of -3.32 . Although amplification efficiencies of between $90 \%$ and $110 \%$ are considered to be acceptable, a small change in PCR efficiency from 100 to $97 \%$ over 30 cycles equates to a 57\% difference in input DNA calculation, while a change from 100 to $90 \%$ over the same range makes a $365 \%$ difference (Boulter et al. 2016). The $r^{2}$ and the standard error of the estimated amplification efficiency can be used to evaluate the quality of the efficiency determination, while the intercept of the standard curve on the y-axis gives a theoretical sensitivity of the assay, denoting the number of cycles required for the detection of a single unit of measurement. The quantities of target sequences in all samples can be calculated by comparing the respective $\mathrm{Cq}$ measurement to the corresponding standard curve calibration model. A standard curve can be constructed using a variety of control materials including plasmid DNA constructs, PCR amplicons, synthetic RNA or DNA, genomic DNA, cDNA, RNA or DNA from biological samples, and quantities may consist of certified, reference or information values (Bustin et al., 2009; Hou et al., 2010).

Although RT-qPCR offers a sensitive and specific technique for quantification of nucleic acid targets, reproducibility and reliability are critical and must be established for each assay. 
Variations in protocols, reagents, sample quality, instruments, operators, analysts, data analysis and interpretation across laboratories can lead to the production of inaccurate quantitative data. To circumvent these issues, Bustin and colleagues (2009) recommended a set of protocols and guidelines, "The Minimum Information for Publication of Quantitative Real-Time PCR Experiments (MIQE) for conducting and reporting qPCR data". The guidelines describe experimental stringency and uniformity practices to ensure the production of reproducible and scientifically defensible quantitative data within and between laboratories. Nevertheless, there remain concerns regarding the quality of RT-qPCR results in the published literature (Bustin and Nolan, 2017; Taylor et al., 2019), particularly in the emerging wastewater surveillance literature (Ahmed et al., 2020b).

Herein, we review the SARS-CoV-2 wastewater surveillance literature to assess the appropriate use of RT-qPCR calibrators and associated performance parameters. All publications included in the analysis are compiled in an open access database as detailed below.

\section{Screening the SARS-CoV-2 Wastewater Surveillance Literature}

We screened a total of 125 preprint and peer-reviewed publications pertaining to wastewater surveillance for SARS-CoV-2 RNA as listed on the COVID-19 Wastewater-based Epidemiology Collaborative (WBEC) publication map (https://www.covid19wbec.org/publication-map) on 15 May 2021. Of those publications, 44 were excluded from further analysis due to use of platforms other than RT-qPCR for SARS-CoV-2 RNA quantification (e.g., digital PCR), reporting qualitative results (e.g., positive/negative or Cq values), or genomic rather than quantitative analysis. The remaining 81 publications (46 peer-reviewed, 35 pre-prints) reported quantitative results (GC) as measured by RT-qPCR. Where available, we extracted the following information for each RT-qPCR assay reported: SARS-CoV-2 gene target, one-step or two-step RT-qPCR 
protocol, standard curve parameters (y-intercept, slope, $r^{2}$, efficiency), limits of detection and quantification, the dynamic range, control material used for standard curve (i.e., plasmid DNA, gBlock, PCR amplicon, etc.), the vendor of the control material, if pre-treatment of the control material was performed, and if independent quantification was performed prior to use. The included studies were divided evenly among three co-authors. Relevant data were extracted with duplicated review of 5 to 10 randomly selected entries by each author. The resulting database was independently reviewed by each author and discrepancies remedied by discussing to form a consensus. The consensus database was then independently reviewed and audited by a fourth author. The compiled database we used for the analysis can be found at https://osf.io/q7dnp/ doi: 10.17605/OSF.IO/Q7DNP. Our purpose in conducting this review is not to identify individual laboratories with questionable practices, but to highlight the importance of reporting standard curve parameters and to assess the performance of the wastewater surveillance community as a whole. For this reason, we have not provided citations when discussing individual publications.

\section{RT-qPCR and Standard Curve Reporting}

From the 81 selected publications, we extracted details pertinent to the assays used, standard curve parameters, and positive control materials for 208 separate quantitative assays (i.e., an average of 2.6 assays per publication). Only $26 \%$ of the total 208 RT-qPCR assays reported in the literature included all essential standard curve parameters, with $41 \%$ (86 of 208) reporting at least one standard curve parameter, i.e., 30, 33, 35, and 40\% of studies detailing y-intercept, slope, $r^{2}$ values, and efficiency data, respectively. Among the 208 RT-qPCR assays, 130 targeted the $\mathrm{N}$ gene, 25 targeted ORF1, 23 targeted the E gene, 19 targeted RdRp, and 10 targeted the $\mathrm{S}$ gene, while one did not report any assay target. For assays targeting the $\mathrm{N}$ gene of SARS-CoV-2, the CDC N1 assay was applied most frequently (39\%) followed by the CDC N2 assay (32\%). 
Together, these two assays accounted for $45 \%$ of the RT-qPCR assays reportedly used to quantify SARS-CoV-2 RNA in wastewater.

RT-qPCR assays are performed using two approaches: a separate RT reaction (i.e., cDNA synthesis) followed by qPCR (two-step) or a combined RT and qPCR reaction in the same tube (one-step). The two-step approach often uses random hexamer primers during RT followed by target-specific primers with the qPCR step offering more flexibility to optimize amplification conditions (Bustin and Nolan, 2017). But the two-step approach requires additional sample handling potentially leading to greater measurement variability and risk of contamination. Conversely, the one-step approach utilizes gene-specific primers and minimizes sample handling by carrying out the RT and qPCR steps in the same microtube, reducing bench time and risk of contamination at the expense of less flexibility for assay optimization.

The majority ( $83 \%$ ) of the reported RT-qPCR assays utilized the one-step protocol and 13\% of RT-qPCR assays were performed as two-step assays. The remaining $4 \%$ did not report whether a one-step or two-step protocol was used. The performance of one-step and two-step RT-qPCR assays were compared in a previous study and the results indicated significant variation in quantification of the targets between the two protocols (Bustin and Nolan, 2017). However, in an earlier study (Wackerd and Godard, 2005), both one-step and two-step protocols produced similar standard curves with RT-qPCR efficiencies close to $100 \%$ suggesting that discrepancies may be protocol and assay specific.

\section{CDC N1 and N2 Standard Curves}

To assess the impact of heterogeneity in reported RT-qPCR standard curves used for quantification of SARS-CoV-2 RNA in wastewater, we analyzed the reported y-intercepts, slopes, $r^{2}$ values, and efficiencies for the two most frequently used RT-qPCR assays, CDC N1 
and N2 (Figure 1). For review, the copy number in an unknown sample is calculated from the standard curve y-intercept and slope per equation (1).

Copy Number $=\frac{C q-Y_{\text {intercept }}}{\text { slope }}$

\subsection{Y-intercept values}

Reported y-intercepts for CDC N1 $(n=21)$ and N2 $(n=18)$ standard curves (Figure 1A) ranged from 36.1 to 42.5 and 37.8 to 53.5 , respectively, with 30 and 24 publications, respectively not reporting this value. These intercepts indicate that anywhere from 36 to 43 RT-qPCR thermal cycles would be required to detect a single GC by the CDC N1 assay and 38 to 54 for the CDC N2 assay. However, these values were obtained using a variety of reference materials from different vendors and the y-intercept value depends on the concentration or copy number associated with that reference material. So, for example, starting with a nominally high copy number standard results in a lower y-intercept value compared with the same reference material that has been ascribed a lower copy number value. The most common source for standard material was plasmid from IDT ( $n=13$ and 11 for N1 and N2, respectively) and y-intercepts reported range from 36.1 to 42.5 (N1) and 37.8 to 53.5 (N2). Considering these are reported to be the same standard, presumably quality controlled prior to shipment and documented with the same ostensible copy number, this draws attention to the subsequent handling of this reference material. 


\subsection{Slope values and RT-qPCR efficiencies}

qPCR efficiency values are derived from the slope of a standard curve per equation (2) and MIQE guidelines specify an acceptable range of $90 \%$ to $110 \%$ (A-Z of Quantitative PCR, 2004; Bustin et al. 2009).

$E=10^{[-1 / \text { slope }]}-1$

While efficiency values often vary between templates, they are typically highly reproducible for the same template (A-Z of Quantitative PCR, 2004).

The ideal slope of a standard curve is -3.32, which indicates $100 \%$ RT-qPCR efficiency, although a range from $-3.1(110 \%)$ to $-3.58(90 \%)$ is typical for an optimized probe-based assay (A-Z of Quantitative PCR, 2004). Slope is also typically more reproducible between laboratories and instruments than the y-intercept (A-Z of Quantitative PCR, 2004). Reported RT-qPCR slopes for CDC N1 and N2 assays are displayed in Figure 1B. For the CDC N1 assay, the mean standard curve slope was -3.29 , which is within the typical interval for a probed-based assay; however, the reported slopes ranged from -3.60 (90\%) to -2.40 (161\%); outside the acceptable range of $-3.1(110 \%)$ to $-3.58(90 \%)$. A similar pattern is seen for CDC N2 with a mean slope of $-3.46(95 \%)$ and a range from $-4.48(67 \%)$ to $-2.72(133 \%)$.

Reported and calculated RT-qPCR efficiencies (calculated with reported slope if not explicitly described in the publication) for the CDC N1 and N2 assays are shown in Figure 1D. For reported CDC N1 standard curves, the mean efficiency was 101\% (median 95.8\%) and ranged from 89.6 to $161 \%$. For CDC N2 assays the range was from 65 to $129 \%$, with a mean efficiency of $95.8 \%$ (median 95\%). 


\section{$4.3 r^{2}$ values}

Standard curves should demonstrate strong linear fits with $r^{2}$ values usually ranging from 0.980 to 1.00 (A-Z of Quantitative PCR, 2004). The $r^{2}$ value of a standard curve is influenced by the precision of replicate standard material Cq measurements. Lower $r^{2}$ values indicate contributions to variation from sources other than the control material copy number (e.g., pipetting error, standard dilution preparation error). For CDC N1 and N2 assays as shown in Figure $1 \mathrm{C}, r^{2}$ values ranged from 0.700 to 1.00 and 0.900 to 1.00 , respectively. While means were comparable $(\mathrm{N} 1=$ $0.973, \mathrm{~N} 2=0.989)$, the CDC N1 assay exhibited a much wider range of reported $r^{2}$ values. The lowest reported $r^{2}$ value across all SARS-CoV-2 RT-qPCR assays were 0.700 for a CDC N1 standard curve. This value is well below 0.980 and strongly suggests variation attributable to sources other than the standard material GC number.

\subsection{Within-Study Variation of Standard Curve Parameters}

MIQE guidelines recommend reporting the confidence interval or standard error of the RT-qPCR efficiency, but do not deem this practice essential (Bustin et al. 2009). Standard deviations were reported for all standard curve parameters for only seven RT-qPCR assays in three publications of SARS-CoV-2 quantification in wastewater. Ranges of at least one standard curve parameter were reported for another 12 assays, bringing the total to $9 \%$ of RT-qPCR assays with some reporting of variation. No study specified whether the reported variation was a measure of repeatability (within run variation) or reproducibility (between run variation). 

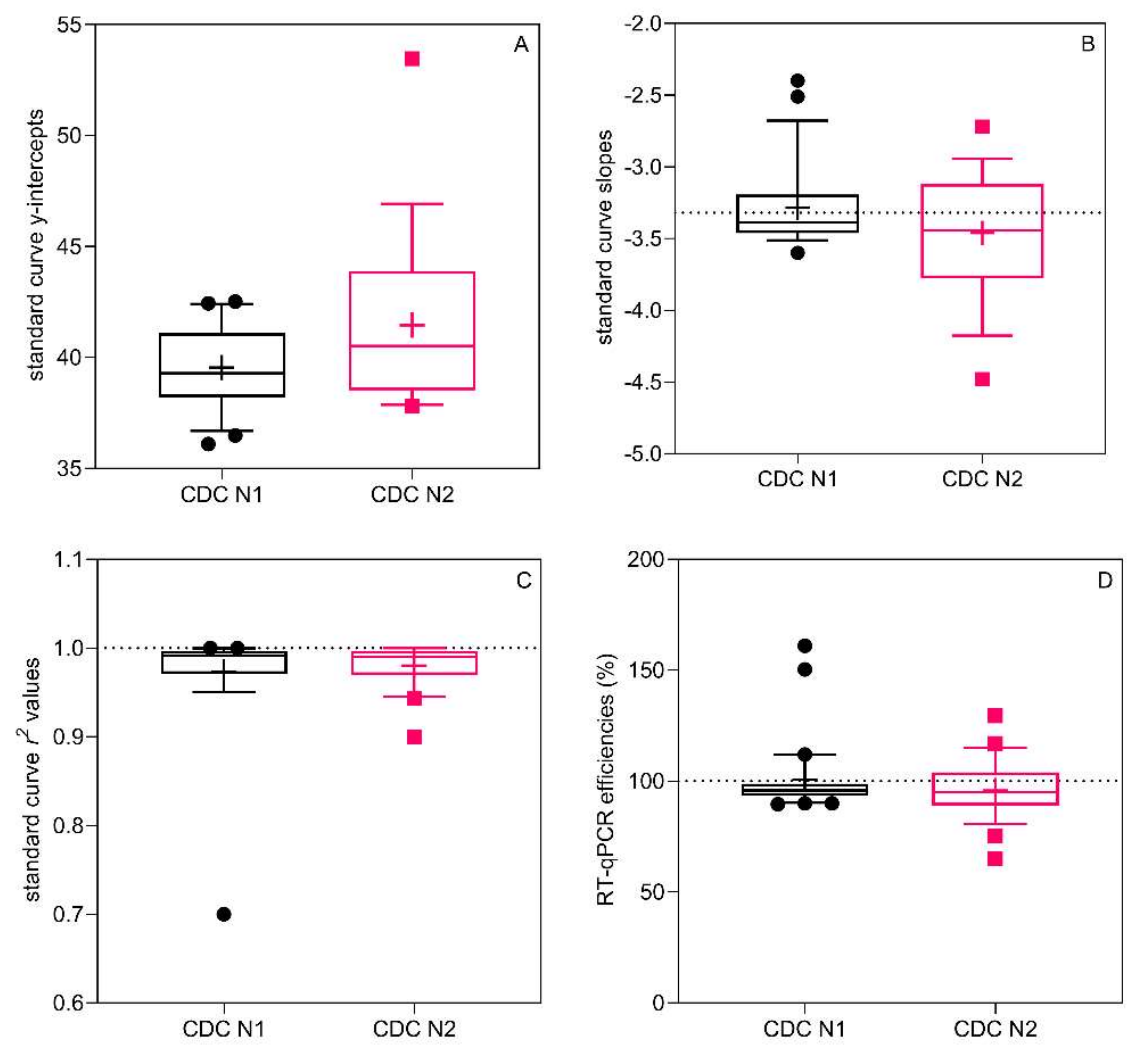

Figure 1. SARS-CoV-2 CDC N1 and N2 standard curve parameters as reported in the literature: (A) y-intercept values, (B) slopes, (C) $r^{2}$ values, and (D) RT-qPCR efficiencies. The boxplots display the $10^{\text {th }}$ and $90^{\text {th }}$ percentiles (whiskers), interquartile range (box), median (line), and mean $(+)$. The dashed line on panel (B) depicts an ideal slope of -3.32 , on panel (C) depicts an $r^{2}$ value of 1.00, and on panel (D) depicts an ideal efficiency of $100 \%$.

\section{Standard Curve Control Materials}

For RT-qPCR, performance is dependent on the standard materials used to produce the standard curve as well as good laboratory practices (A-Z of Quantitative PCR, 2004). Careful, application-specific optimization is required to maximize the performance of the standard materials and the subsequent quantification of genetic targets in samples. 
In many cases, commercially available control materials were employed. Often these materials are provided at a vendor-specified titer, which is assumed to increase the likelihood of producing reliable standard curves. However, it is clear from the variability described above that either the dilutions or the pipetting steps are highly variable and operator-dependent, something that has been shown to be the case (Bustin, 2002).

\subsection{Control Material Reporting}

A description of the control material used was reported for $78 \%$ of the SARS-CoV-2 RT-qPCR assays in the wastewater surveillance literature. Although, the description was sometimes ambiguous including terms such as "genes encoding nucleocapsid protein", "synthetic oligonucleotide", and "cDNA standards". Such ambiguous descriptions can be especially problematic if the vendor is not specified, which was the case for $28 \%$ of the reported assays. Using broad classifications, control materials included plasmids for 59 assays, synthetic cDNA for 29, synthetic RNA for 36, various forms of transcripts for 19, ambiguous or unclear for 19, and not reported for 46 RT-qPCR assays. Plasmids and synthetic oligonucleotides (cDNA or RNA) accounted for the majority of reported control materials with a nearly even split between the two (28 and 31\%, respectively). Pre-treatment of control materials was reported for 7 RTqPCR assays (heat inactivation of isolated strain in two and linearization of plasmid in the remaining five instances). Independent quantification of control materials was only reported for $6 \%$ (12 of 208) of RT-qPCR assays. 


\subsection{Effects of Control Material Type on Standard Curve Parameters}

Previous studies have reported significant variations in $\mathrm{Cq}$ for standard curves produced using non-linearized plasmids compared to linear control materials (synthetic cDNA or RNA) (Hou et al., 2010; Lin et al., 2011; Werling et al., 2015; Beinhauerova et al., 2020), although this is not universal findings (Oldham et al., 2012). Chik et al. (2021) reported differences up to one order of magnitude between laboratories using linear vs. plasmid standards with an $8.4 \mathrm{Cq}$ difference observed in one instance between linearized versus non-linearized plasmids. Gerrity et al. (2020) reported a droplet digital PCR (ddPCR)-measured copy number 5-fold lower than the vendorspecified titer for a circular plasmid control and noted heteroscedasticity in the variance caused by the non-linearized plasmid control material with a 9.4-fold bias at $40 \mathrm{Cq}$ and a 5.5 -fold bias at 25 Cq. Others have noted that circular DNA plasmids may require linearization to avoid impacts to PCR efficiency leading to subsequent bias in qPCR measurements (Hou et al. 2010).

Linearization was only reported for $8 \%$ of the RT-qPCR assays where a plasmid was used as the control material for standard curves.

We considered the effects of control material type, linear materials (cDNA or RNA) versus plasmid materials (mostly circular), on reported RT-qPCR standard curves for the CDC N1 and N2 assays across the wastewater surveillance literature. From the literature, we were able to extract standard curve parameters for $21 \mathrm{CDC}$ N1 assays (seven linear control materials and 14 plasmid), and 18 CDC N2 assays (six linear control materials and 12 plasmid). Figure 2 shows scatter plots of each standard curve parameter for CDC N1 and CDC N2 assays stratified by control material type. Standard curve performance parameters based on synthetic RNA or CDNA and plasmid materials were evaluated via a Mann-Whitney $U$ test (Mann and Whitney, 1947). A 
significant difference was only observed between the standard curve slopes and efficiencies produced for the CDC N1 assays ( $p=0.0402$; Figure 2B, $p=0.0181$; Figure 2D, respectively).
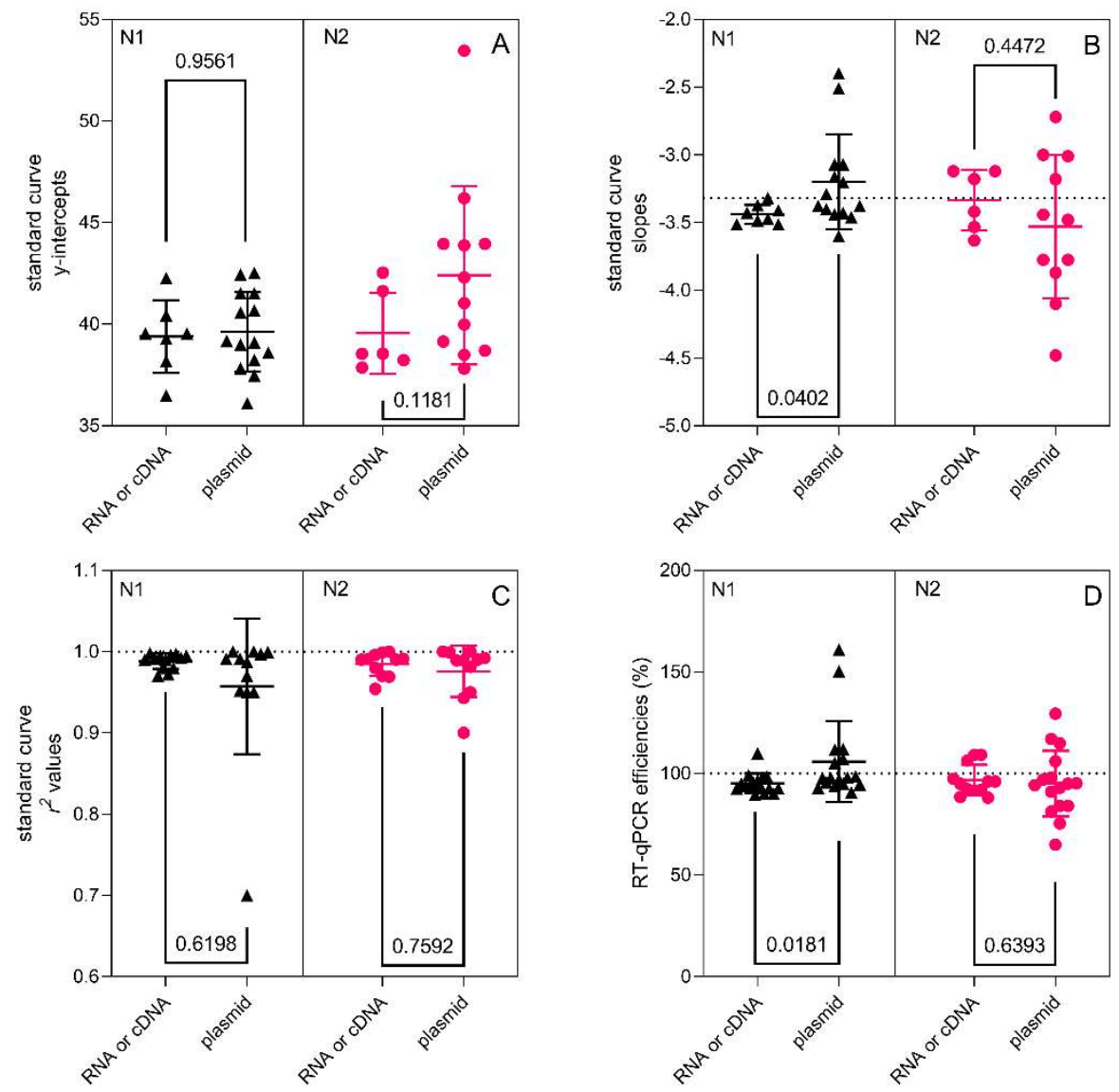

Figure 2. Dot plots of reported CDC N1 and N2 RT-qPCR standard curve parameters: (A) yintercepts, (B) slopes, (C) $r^{2}$ values, and (D) efficiencies stratified by linear synthetic RNA or cDNA versus plasmid control material. Individual values, mean, and standard deviation are displayed. The dashed line on panel (B) depicts an ideal slope of -3.32 , on panel (C) depicts an $r^{2}$ value of 1.00, and on panel (D) depicts an ideal efficiency of $100 \%$. 
Although most of the statistical tests imply no significant differences, the scatter plots in Figure 2 suggest that plasmid control materials consistently exhibit a broader range of variation across all standard curve parameters for both the CDC N1 and N2 assays. A coefficient of variation (CV = standard deviation/mean) was calculated for each standard curve parameter and standard material group combination for both CDC N1 and N2 assays, CV values were greater for curves produced using plasmid materials compared to linearized materials, further supporting this observation. Differences were particularly pronounced for standard curve slopes, $r^{2}$ values, and RT-qPCR efficiencies. For the CDC N2 assay, the use of plasmid control materials resulted in a roughly 2fold increase in the $\mathrm{CV}$ of the standard curve slope, $r^{2}$ value, and efficiency. Whereas, for the CDC N1 assay the plasmid control materials resulted in an 5-fold, 9-fold, and 4-fold increase in CV for the slope, $r^{2}$ value, and efficiency, respectively. These observations suggest increased variability, and therefore, lower reproducibility, among CDC N1 and N2 standard curves produced using non-linearized plasmid controls materials compared to linear RNA and cDNA materials with the effect being particularly pronounced for the CDC N1 RT-qPCR assay. As shown in Figure 3, we further visualized the effect of this variation by fitting linear regressions to reported RT-qPCR standard curves for the CDC N1 and N2 assays stratified by control material treating each curve as a replicate. While the mean slopes (m) and y-intercepts (b) are comparable, the increased width of the shaded area illustrates the increased $99^{\text {th }}$ percentile prediction interval for the standard curves produced from plasmids compared to synthetic RNA or cDNA. The $r^{2}$ values for both CDC N1 and N2 plasmid-based standard curves ( 0.943 and 0.897, respectively) are lower than the $r^{2}$ values for RNA/cDNA curves and are well below the MIQE guideline recommended 0.980 lower bound. These decreased $r^{2}$ values suggest an increasing proportion of the variation in experiments performed with plasmids is attributable to a 
source other than variability in standard dilution GC quantities. Our observations corroborate those of Chik et al. (2021) and Gerrity et al. (2020) and further suggest a systematic deficiency in the protocols and procedures being used for plasmid control materials in RT-qPCR quantification of SARS-CoV-2 RNA in wastewater.
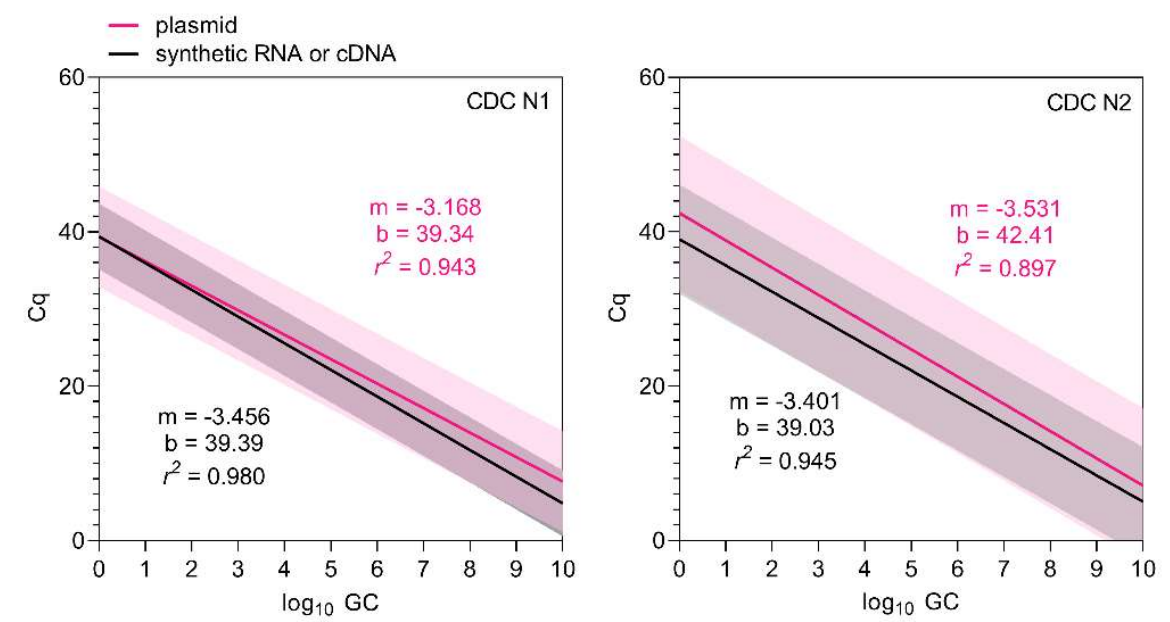

Figure 3. Linear regressions fit to the reported standard curves for CDC N1 assays (left; $n=22)$ and CDC N2 assays (right; $n=19$ ) in the wastewater surveillance literature. Regressions fit to data from synthetic RNA or cDNA control materials are denoted in black, while those fit to plasmid control materials are denoted in pink. The shaded region displays the $99^{\text {th }}$ percentile for each. The slope (m), y-intercept (b), and $r^{2}$ values for the linear fit to each set of data are shown in the color corresponding to their regression line.

\section{Reported LODs and LOQs}

The MIQE guidelines list "evidence for limit of detection" (LOD) and "Cq variation at lower limit” (LOQ) as of essential importance for reporting RT-qPCR experiments. LOD values were reported for only $34 \%$ of RT-qPCR assays, ranging between one and $500 \mathrm{GC}$ per reaction (the 
most frequently used unit). LOD values were also reported in volumetric units (i.e., $\mu \mathrm{L}, \mathrm{mL}$, etc.), but the source of this volume (e.g., sewage, extract eluate, reaction mixture) was often unspecified. A method definition for the LOD determination was rarely provided and, in several cases, the reported LOD values were below the 95\% LOD theoretical limit of $3 \mathrm{GC} /$ reaction derived from the Poisson distribution (Bustin et al., 2009). An LOQ was reported for only 19\% of RT-qPCR assays, about half as frequently as an LOD. LOQ values were also most frequently reported in GC per reaction with values ranging from 5 to more than 10,000. Similar to LOD, when volumetric units were used to report LOQ, the source of the relevant volume was often ambiguous. Furthermore, when multiple RT-qPCR assays were used in a single publication, reported LOD and LOQ values were often not linked to a corresponding assay.

\section{Discussion}

The quantitative potential of RT-qPCR assays has resulted in the broadening of its use from qualitative detection of SARS-CoV-2 to the quantification of viral load in patients as well as quantifying the virus in environmental samples. While a binary result demands reliability, specificity and sensitivity, quantitative analysis sets a much greater bar in terms of assay quality as conveyed by reproducibility, accuracy, precision, and comparability. These parameters are revealed using optimised assay-specific standard curves established using appropriate and validated control materials (A-Z of Quantitative PCR, 2004). The MIQE guidelines, published 11 years ago, are crafted to guide investigators and reviewers in their production of reliable and reproducible RT-qPCR data (Bustin et al., 2009). Such reliability is even more critical when the data produced are used to guide responses to an outbreak or pandemic. In response to the ongoing COVID-19 pandemic, many laboratories, some with limited or no environmental microbiology experience, quickly pivoted and adopted various assays, protocols, materials, and 
techniques, including RT-qPCR to detect and quantify SARS-CoV-2 RNA in wastewater. While such efforts are commendable and shortcomings under the duress of standing up entire surveillance programs on short notice are understandable, this review suggests a neglect of best practices pertaining to RT-qPCR experiments in the SARS-CoV-2 wastewater surveillance literature. Consequently, there is significant potential for inaccurate and misleading reporting of SARS-CoV-2 with wide-ranging implications for the development of policies and guidelines. Furthermore, the departure from best practice in this important process has serious implications for environmental monitoring for high priority biothreat agents in general.

Basic and essential information for RT-qPCR assays including the standard curve parameters of y-intercept, slope and/or efficiency, and $r^{2}$ value were reported for only $26 \%$ of the RT-qPCR assays used for wastewater surveillance. Variation in these parameters is even more rarely reported being published for only $9 \%$ of assays. The reported standard curve data exhibit broad heterogeneity that, in turn, limits the reproducibility and reliability of RT-qPCR data for SARSCoV-2 wastewater surveillance. For example, y-intercepts ranged from 36.1 to 53.5; slopes ranged from -2.4 to $-4.5 ; r^{2}$ values were as low as 0.700 with reported efficiencies ranging from 65 to $161 \%$. Many of these are well outside the bounds of expected performance for optimized RT-qPCR assays. Variation in RT-qPCR performance seems further exacerbated by the prevalent use of a plasmid control material without linearization. This practice is likely to produce increased variation across all standard curve parameters with a particularly pronounced increase in the widely used CDC N1 RT-qPCR assay.

The published SARS-CoV-2 wastewater surveillance literature also contains other scenarios that reinforce the need for higher standards in practice and reporting. In some publications, "standard curves" were generated using one to three standard dilutions, while others made GC 
concentration estimates in samples falling well outside the defined dynamic range. Many publications provided no standard curve information at all. Another publication stated that the quantitative data were generated by a commercial lab and did not provide any pertinent RTqPCR information, not even the assay used to generate the data. In a few cases, sophisticated epidemiological models such as susceptible-exposed-infectious-recovered (SEIR), vector autoregression, and Monte Carlo simulations were applied to quantitative data from RT-qPCR experiments with no standard curve parameters reported, making it essentially impossible to validate the utility of these approaches.

There are important limitations to this review. First, this is not a formal systematic review, instead publications were identified from a curated collection of SARS-CoV-2 wastewater surveillance publications. Although this review did not consider every SARS-CoV-2 RT-qPCR publication, the inclusion of 125 works proved sufficient to identify multiple deficiencies in standard curve performance and reporting practices. Second, this review relied on self-reported data from pre-print and published scientific literature, which could be subject to bias.

Additionally, it is important to note that variation between RT-qPCR replicates, experimental runs, instruments, and laboratories is expected to a degree, and is well documented for various SARS-CoV-2 RT-qPCR assays (Bustin et al., 2021). While it is not possible to independently account for this expected variation, the variability observed in this review often exceeded recommend best practices strongly suggesting that the wastewater surveillance community must make a concerted effort to improve both reporting of RT-qPCR parameters and optimization of SARS-CoV-2 RT-qPCR assays. 


\section{Conclusions and Recommendations}

This brief review of the literature describing the use of RT-qPCR assays for monitoring wastewater has revealed significant variation in the standards of the reported results, although the paucity of quality control data makes it difficult to ascertain which reports are likely to be the most credible. Hence there is an urgent need to improve the transparency of reporting as well as disclose more information about the characteristics of the assays being used. We propose the following measures:

- A starting point for improvement would be the adoption of the MIQE guidelines for reporting RT-qPCR (and digital PCR) experiments performed for wastewater surveillance purposes (Bustin et al., 2009; Huggett et al., 2013). Journal editors and reviewers should consider requiring the submission of the pertinent MIQE checklist (https://rdml.org/miqe.html) for all SARS-CoV-2 wastewater surveillance publications.

- To produce reliable data for public health decision-making, the wastewater surveillance community should, at a minimum, aspire to achieve $100 \%$ reporting of all standard curve performance parameters (y-intercept, slope/efficiency and, $r^{2}$ value). If standard curve parameters do not fall within general data acceptance ranges, additional actions may be required such as optimization, calibration of pipets and/or thermal cycle instrumentation, among others until the appropriate performance is achieved.

- Because the reproducibility of RT-qPCR data relies, in part, on the standard curves produced by control materials, the type and vendor for such materials should be completely and unambiguously reported. Any pre-treatment or manipulation of these control materials prior to quantification should also be documented, especially for plasmid controls. Caveat emptor must be the motto when relying upon commercially available control materials. 
- It would also be useful to independently confirm vendor-reported titers to ensure that materials were not degraded during shipment or due to mishandling in the laboratory. In summary, to maximize the utility of wastewater surveillance for public health, it is time to "walk the walk" (Bustin and Nolan, 2017).

\section{Disclaimer}

Information has been subjected to U.S. EPA peer and administrative review and has been approved for external publication. Any opinions expressed in this paper are those of the authors and do not necessarily reflect the official positions and policies of the U.S. EPA. Any mention of trade names or commercial products does not constitute endorsement or recommendation for use. 


\section{References}

1. Ahmed, W., Tscharke, B., Bertsch, P.M., Bibby, K., Bivins, A., Choi, P., Clarke, L., Dwyer, J., Edson, J., Nguyen, T.M.H., O’Brien, J.W., Simpson, S.L., Sherman, P., Thomas, K.V., Verhagen, R., Zaugg, J., Mueller, J.F., 2021a. SARS-CoV-2 RNA monitoring in wastewater as a potential early warning system for COVID-19 transmission in the community: A temporal case study. Sci Total Environ. 761, 144216.

2. Ahmed, W., Bivins, A., Bertsch, P.M., Bibby, K., Choi, P.M., Farkas, K., Gyawali, P., Hamilton, K.A., Haramoto, E., Kitajima, M., Simpson, S.L., Tandukar, S., Thomas K.V., Mueller, J.F., 2020b. Surveillance of SARS-CoV-2 RNA in wastewater: Methods optimization and quality control are crucial for generating reliable public health information. Curr Opin Environ Sci Health. 17, 82-93.

3. Ahmed, W., Simpson, S., Bertsch, P., Bibby, K., Bivins, A., Blackall, L., Bofill-Mas, S., Bosch, A., Brandao, J., Choi, P., Ciesielski, M., Donner, E., D'Souza, N., Farnleitner, A., Gerrity, D., Gonzalez, R., Griffith, J., Gyawali, P., Haas, C., Hamilton, K., Hapuarachchi, C., Harwood, V., Haque, R., Jackson, G., Khan, S., Khan, W., Kitajima, M., Korajkic, A., La Rosa, G., Layton, B., Lipp, E., McLellan, S., McMinn, B., Medema, G., Metcalfe, S., Meijer, W., Mueller, J., Murphy, H., Naughton, C., Noble, R., Payyappat, S., Petterson, S., Pitkanen, T., Rajal, V., Reyneke, B., Roman, F., Rose, J., Rusinol, M., Sadowsky, M., Sala-Comorera, L., Setoh, Y.X., Sherchan, S., Sirikanchana, K., Smith, W., Steele, J., Sabburg, R., Symonds, E., Thai, P., Thomas, K., Tynan, J., Toze, S., Thompson, J., Whiteley, A., Wong, J., Sano, D., Wuertz, S., Xagoraraki, I., Zhang, Q., Zimmer-Faust, A., Shanks, O., 2021b. Minimizing errors in RT-PCR detection and quantification of SARS-CoV-2 RNA for wastewater surveillance. Preprints. 2021040481. doi: 10.20944/preprints202104.0481.v1.

4. Beinhauerova, M., Babak, V., Bertasi, B., Boniotti, M.B., Kralik, P., 2020. Utilization of digital PCR in quantity verification of plasmid standards used in quantitative PCR. Front Mol Biosci. 7, 155.

5. Bivins, A., North, D., Ahmad, A., Ahmed, W., Alm, E., Been, F., Bhattacharya, P.,Bijlsma, L., Boehm, A.B., Brown, J., Buttiglieri, G., Calabro, V., Carducci, A., Castiglioni, S., Gurol, Z.C., Chakraborty, C., Costa, F., Curcio, S., de los reyes III, F.L., Delgado Vela, J., Farkas, K., Fernazdez-Casi, X., Gerba, C., Gerrity, D., Girones, R., Gonzzalez, R., Haramoto, E., Harris, A., Holden, P.A., Ispam, M.T., Jones, D.L., Kasprzyk-Hordern, B., Kitajima, M., Kotlarz, N., Kumar, M., Kuroda, K., La Rosa, G., Malpei, F., Mautus, M., Mclellan, S.L., Medema, G., Meschke, J.S., Mueller, J., Newton, R.J., Noble, R.T., van Nuijs, A., Peccia, J., Perkins, T.A., Pickering, A.J., Rose, J., Sanchez, G., Smith, A., Stadler, L., Stauber, C., Thomas, K., van der Voorn, T., Wiggington, K., Zhu, K., Bibby, K., 2020. Wastewater-based epidemiology: global collaborative to maximize contributions in the fight against COVID-19. Environ. Sci. Technol. 54(13), 7754-7757.

6. Bustin, S.A. 2002. Quantification of mRNA using real-time reverse transcription PCR (RTPCR): trends and problems. J. Mol. Endocrinol. 29:23-39.

7. Bustin, S.A., Benes, V., Garson, J.A., Hellemans, J., Huggett, J.F., Kubista, M., Mueller, R.D., Nolan, T., Pfaffl, M.W., Shipley, G.L., Vandesompele, J., Wittwer, C.T., 2009. The MIQE guidelines: Minimum information for publication of quantitative real-time PCR experiments. Clin. Chem. 55, 611-622. 
8. Bustin, S., Nolan, T., 2017. Talking the talk, but not walking the walk: RT-qPCR as a paradigm for the lack of reproducibility in molecular research. Eur J Clin Invest. 47, 756774.

9. Bustin, S., Mueller, R., Shipley, G., Nolan, T., 2021. COVID-19 and diagnostic testing for SARS-CoV-2 by RT-qPCR- Facts and Fallacies. Int J Mol Sci. 22, 2459.

10. Boulter, N., Suarez, F.G., Schibeci, S., Sunderland, T., Tolhurst, O., Hunter, T., Hodge, G., Handelsman, D., Simananien, U., Hendriks, E., Duggan, K., 2016. A simple, accurate and universal method for quantification of PCR. BMC Biotechnol. 16,27.

11. Chik, A.H.S., Glier, M.B., Servos, M., Mangat, C.S., Pang, X.-L., Qiu, Y., D’Aoust P.M., Burnet, J.-B., Delatollea, R., Dorner, S., Geng, Q., Giesy Jr., J.P., McKay, R.M., Mulvey, M.R., Prystajecky, N., Srikanthan, N., Xie, Y., Conant, B., Hrudey, S.E., 2021. Canadaian SARS-CoV-2 Inter-laboratory Consortium. Comparison of approaches to quantify SARSCoV-2 in wastewater using RT-qPCR: Results and implications from a collaborative interlaboratory study in Canada. J. Environ. Sci. (China). 107, 218-229.

12. D'Aoust P.M., Graber, T.E., Mercier E., Montpetit, D., Alexandrov, I., Neault, N., Baig A.T., Mayne, J., Zhang, X., Alain, T., Servos, M.R., Srikanthan, N., MacKenzie, M., Figeys, D., Manuel, D., Jüni, P., MacKenzie, A.E., Delatolla, R., 2021. Catching a resurgence: Increase in SARS-CoV-2 viral RNA identified in wastewater $48 \mathrm{~h}$ before COVID-19 clinical tests and $96 \mathrm{~h}$ before hospitalizations. Sci. Total Environ. 770:145319.

13. Gerrity, D., Papp, K., Stoker, M., Sims, A., Frehner, W., 2020. Early-pandemic wastewater surveillance of SARS-CoV-2 in Southern Nevada: Methodology, occurrence, and incidence/prevalence considerations. Water Res X, 10, 100086.

14. Hou, Y., Zhang, H., Miranda, L., Lin, S., 2010. Serious overestimation in quantitative PCR by circular (supercoiled) plasmid standard: microalgal pcna as the model gene. PLoS One. 2010;5:e9545.

15. Huggett, J.F., Foy, C.A., Benes, V., Emslie, K., Garson, J.A., Haynes, R., Hellemans, J., Kubista, M., Mueller, R.D., Nolan, T., Pfaffl, M.W., Shipley, G.L., Vandesompele, J., Wittwer, C.T., Bustin, S.A., 2013. The digital MIQE Guidelines: Minimum Informantion for publication of quantitative digital PCR experiments. Clin. Chem. 59, 892-902.

16. Kantor, R.S., Nelson, K.L., Greenwald, H.D., Kennedy, L.C., 2021. Challenges in measuring the recovery of SARS-CoV-2 from wastewater. Environ. Sci. Technol. 55:3514-3519.

17. Li, B., Di, D.Y.W., Saingam, P., Jeon M.K., Yan, T., 2021. Fine-scale temporal dynamics of SARS-CoV-2 RNA abundance in wastewater during a COVID-19 Lockdown. Water Res. $197,117093$.

18. Lin, C.H., Chen, Y.C., Pan, T.M., 2011. Quantification bias caused by plasmid DNA conformation in quantitative real-time PCR assay. PLoS ONE. 6(12), e29101.

19. Mann, H.B., Whitney, D.R., 1947. On a test of whether one of two random variables is stochastically larger than the other. Ann. Math. Statist. 18(1): 50-60.

20. Medema, G., Heijnen, L., Elsinga, G., Italiaander, R., 2020a. Presence of SARSCoronavirus-2 RNA in sewage and correlation with reported COVID-19 prevalence in the early stage of the epidemic in the Netherlands. Environ. Sci. Technol. Lett. 7 (7), 511-516.

21. Medema, G., Been, F., Heijnen, L., Petterson, S., 2020b. Implementation of environmental surveillance for SARS-CoV-2 virus to support public health decisions: opportunities and challenges. Curr. Opin. Environ. Sci. Health. 17, 49-71. 
22. Michael-Kordatou, I., Karaolia, P., Fatta-Kassinos, D., 2020. Sewage analysis as a tool for the COVID-19 pandemic response and management: the urgent need for optimised protocols for SARS-CoV-2 detection and quantification. J. Environ. Chem. Eng. 8(5), 104306.

23. Naughton, C.C., Roman Jr., F.A., Alvarado, A.G.F., Tariqi, A.Q., Deeming, M.A., Bibby, K., Bivins, A., Rose, J.B., Medema, G., Ahmed, W., Katsivelis, P., Allan, V., Sinclair, R., Zhang, Y., Kinyua, M.N., 2021. Show us the data: Global COVID-19 wastewater monitoring efforts, equity, and gaps. MedRXiv. doi: https://doi.org/10.1101/2021.03.14.21253564.

24. Oldham, A.L., Duncan, K.E., 2012. Similar gene estimates from circular and linear standards in quantitative PCR analyses using the prokaryotic 16S rRNA gene as a model. PLoS ONE 7(12), e51931.

25. Peccia, J., Zulli, A., Brackney, D.E. Grubaugh, N.D., Kaplan, E.H., Casanovas-Massana, A., Ko, A.I., Malik, A.A., Wang, D., Warren, J.L., Weinberger, D.N., Arnold, W., Omer, S.B., 2020. Measurement of SARS-CoV-2 RNA in wastewater tracks community infection dynamics. Nat. Biotechnol. 38:1164-1167.

26. Svec, D., Schauberger, A., Novosadova, V., Pfaffl, M.W., Kubista, M., 2015. How good is a PCR efficiency estimate: Recommendations for precise and robust qPCR efficiency assessments? Biomol. Detect. Quant. 3, 9-16.

27. Taylor, S.C., Nadeau, K., Abbasi, M., Lachance, C., Nguyen, M., Fenrich, J., 2019. The Ultimate qPCR experiment: producing publication quality, reproducible data the first time. Trends Biotechnol. 37 (7), 761-774.

28. Wacker, M.J., Godard, M.P., 2005. Analysis of one-step and two-step real-time RT-PCR using SuperScript III. J Biomol Tech.16(3), 266-71.

29. Werling, N.J., Satkunanathan, S., Thorpe, R., Zhao, Y., 2015. Systematic comparison and validation of quantitative real-time PCR methods for the quantitation of adeno-associated viral products. Hum Gene Ther Methods. 26 (3), 82-92.

30. Zhu, Y., Oishi, W., Maruo, C., Saito, M., Chen, R., Kitajima, M., Sano, D., 2021. Early warning of COVID-19 via wastewater-based epidemiology: potential bottlenecks. Sci Total Environ. 767, 145124.

31. A-Z of Quantitative PCR., 2004. Editor: Stephen A. Bustin. Volume 5 of IUL Biotechnology Series, Calif International University Line La Jolla. 